\title{
Weakly frustrated two-dimensional Heisenberg antiferromagnets: thermodynamic properties from a non-perturbative approach
}

\author{
Leonardo Spanu ${ }^{1,3}$ and Alberto Parola ${ }^{2}$ \\ 1 Dipartimento di Fisica "A.Volta", Università di Pavia, I-27100 Pavia, Italy \\ 2 Dipartimento di Fisica e Matematica, Università dell'Insubria, I-22100 Como, Italy \\ 3 International School for Advanced Studies, Via Beirut 4, 34013 Trieste, Italy
}

(Dated: December 14, 2018)

\begin{abstract}
We analyze the thermodynamic properties of the spin- $S$ two-dimensional quantum Heisenberg antiferromagnet on a square lattice with nearest and next-nearest neighbor couplings in the Néel phase $\left(J_{2} / J_{1}<0.4\right)$ employing the quantum hierarchical reference theory (QHRT), a non-perturbative implementation of the renormalization group method to quantum systems. We investigate the staggered susceptibility, the structure factors and the correlation length at finite temperature and for different values of the frustration ratio. From the finite temperature results, we also extrapolate ground state properties, such as spin stiffness and spontaneous staggered magnetization, providing an estimate of the extent of quantum corrections. The behavior of these quantities as a function of frustration may provide some hint on the breakdown of the Néel phase at zero temperature for larger values of $J_{2}$.

PACS numbers: 05.10.Cc75.10.Jm 05.70.Jk 75.40.Cx
\end{abstract}

\section{INTRODUCTION}

Several new magnetic materials characterized by competing (i.e. frustrating) interactions have been recently synthesized ${ }^{1,2}$. Unfortunately, a detailed theoretical understanding of the ground state properties of simple models displaying both frustration and quantum fluctuations is still missing ${ }^{3}$ and the interpretation of the experimental results often relies on simple perturbative or semiclassical estimates. Needless to say, the effects of thermal fluctuations in these systems is far from being understood either, despite the fact that most of the experimentally accessible quantities explicitly refer to the temperature response.

Among the different magnetic models with competing interactions which have been theoretically investigated, the so-called $J_{1}-J_{2}$ model provides a paradigm of the open problems and challenging questions arising in this area of theoretical magnetism ${ }^{3}$. The Hamiltonian of the square lattice Heisenberg antiferromagnet with nearestneighbors (n.n) and next-nearest neighbors interactions (n.n.n.) is

$$
H_{J}=J_{1} \sum_{n . n .} \vec{S}_{\mathbf{i}} \cdot \vec{S}_{\mathbf{j}}+J_{2} \sum_{n . n . n .} \vec{S}_{\mathbf{i}} \cdot \vec{S}_{\mathbf{j}}
$$

where $J_{1}, J_{2}>0$ and $\vec{S}$ are spin- $S$ operators. The model is characterized by a single parameter: $\alpha=J_{2} / J_{1}$ named frustration ratio. The interest in this model was triggered by the discovery of high- $\mathrm{T}_{c}$ superconductors and by the possible connections between the physics of the twodimensional Hubbard model and the $\mathrm{Cu}$ oxides materials. It has been argued that the motion of holes in a quantum antiferromagnet gives rise to effective frustrating couplings in the undoped Heisenberg Hamiltonian ${ }^{4}$, which eventually leads to the breakdown of Néel order. More recently, the physics of the $J_{1}-J_{2}$ model raised a renewed interest due to the discovery of three vanadate compounds $\left(\mathrm{Li}_{2} \mathrm{VOSiO}_{4}, \mathrm{Li}_{2} \mathrm{VOGeO}_{4}\right.$ and $\left.\mathrm{VOMoO}_{4}\right)$ which appear to be characterized by antiferromagnetic nearest and next-nearest neighbor interactions between spin- $1 / 2$ $\mathrm{V}^{4+}$ ions $\mathrm{s}^{2.5}$.

At the classical level, the zero temperature phase diagram of the $J_{1}-J_{2}$ model separates into two regions: for $\alpha<1 / 2$ the system shows Néel order with magnetic wave vector $\mathbf{g}=(\pi, \pi)$, while for $\alpha>1 / 2$ the two sublattice decouple, each displaying Néel order, leading to (infinitely) degenerate classical ground states. This degeneracy is lifted by quantum fluctuations which, at the semi-classical level, select collinear order with periodicity characterized by wave vectors $(\pi, 0)$ or $(0, \pi)$. In recent years the search for a new disordered phase in the intermediate region of frustration $(\alpha \sim 0.5)$, has been the main subject of theoretical investigation ${ }^{6}$ while the thermal properties of frustrated quantum models have attracted comparatively less attention. Finite temperature studies cannot rely on the Monte Carlo simulations, successfully employed in the non-frustrated case: in fact the use of these methods is strongly limited by the wellknown sign problem, a numerical instability which occurs both in fermionic systems and in frustrated bosonic models $\stackrel{7}{\underline{7}}$. On the other hand, the Lanczos-based approaches do not suffer from the sign problem, but the limitations in the cluster size make rather difficult the calculations at finite temperature ${ }^{\frac{8}{}}$. Nevertheless thermodynamic parameters such as spin stiffness and spin velocity have been estimated by exact diagonalization on finite clusters followed by extrapolation to the thermodynamic limit. Unfortunately these small cluster results are always affected by the uncertainties in the finitesize scaling procedure ${ }^{9.10,11}$. Among the numerical approaches, the high temperature expansion method seems to be the natural candidate for the analysis of thermal fluctuations 12 . Indeed, the physics of the collinear phase 
has been recently investigated by several high temperature expansion studies 13.14 . Besides the interest related to the physics of the vanadate materials.5.14, the study of the large $\alpha$-limit of the model was also motivated by the possible observation of a finite temperature 'Ising' transition in the $J_{1}-J_{2}$ model. In fact the residual $Z_{2}$ symmetry of the Hamiltonian in the collinear phase (due to the twofold degeneracy of the ground state) can be broken at finite temperature $\frac{15}{}$, without violating the Mermin-Wagner theorem.

On the analytical side, Ivanov and Ivanov 16 investigated the low-temperature thermodynamics for $0<$ $\alpha<1$, by a modified spin-wave theory (SWT). Using Schwinger-boson mean-field theory Mila et al. examined the spin dynamics of the model, as an effective theory for the high- $\mathrm{T}_{C} \mathrm{Cu}$ oxides ${ }^{17}$. A comprehensive study of the thermodynamics of the collinear phase, within a (semiclassical) effective Hamiltonian approach (PQSCHA), has been performed by Capriotti et al., also for the extreme quantum case $S=1 / 2^{18}$, while only a few investigations have been devoted to the thermodynamics of the weakly frustrated model, in the Néel phase. In the low temperature regime some hint comes from the renormalization group (RG) formalism applied to the non-linear sigma model (NLSM). Within the NLSM approach, the frustrating interaction is treated in mean field approximation and the fluctuations induced by the frustration are in fact neglected. The terms in the Hamiltonian acting on the variables of the same sub-lattice, such as the $J_{2}$ interaction, are integrated out in order to obtain the effective action and then the effect of the frustration reduces to a renormalization of the coupling constant $g^{19}$. As a result, the inclusion of a next nearest neighbor interaction $J_{2}>0$ increases the bare coupling $g$ and ,following the RG picture, enhances short range fluctuations. Indeed, if the frustration is not strong enough to destroy the long range order, the low temperature physics of the systems is believed to be the same of the non-frustrated case, with physical properties,such as spin stiffness and spin velocity, renormalized by the inclusion of frustration. Spin-waves expansion results seems to support this picture. However, even in the non frustrated case, the picture based on NLSM and RG approach does not adequately represent the experimental behavior for the quasi-2D antifferomagnet with $S>1 / 2$. Actually the agreement between theory and experiment seems to hold only for $S=1 / 2$ in the low and intermediate temperature regions. In fact, the effective Hamiltonian description is generally valid in a regime characterized by strong short range correlations and it is not suitable to give a detailed account of the physics in a range of temperature where local order weakens or even disappears.

In order to describe the interplay between frustration, temperature and quantum fluctuations, we follow here a different method which aims to reconcile the RG approach with a microscopic description of the model. This theory, named Quantum Hierarchical Reference Theory $(\mathrm{QHRT})^{20}$ does not rely on a coarse graining procedure leading to some effective action but instead is a way to implement the momentum space renormalization method directly to the physical Hamiltonian. Starting from the mean field approximation, we recursively include the fluctuations over all length scales by means of an evolution equation, which describes how the free energy of the system changes when fluctuations over growing wavelengths are taken into account. The original method developed by Wilson has been largely employed for the investigation of the properties of quantum systems, but, contrary to the weak coupling RG method applied to effective field theories, our approach does not require any mapping on coarse grained actions and applies to all temperature regimes. The resulting evolution equation is formally exact but it is not written in a closed form: An ansatz for the momentum and frequency dependence of the dynamical structure factor is needed in order to put the equation in a closed and solvable form. The choice of a parameterization for the spin-spin correlation function is the only approximation present in this approach.

The paper is organized as follows. In Section $I I$ we derive the evolution equation for the $J_{1}-J_{2}$ model. In Sec. III we investigate the effects of the frustration on the spontaneous magnetization and other ground state properties, like spin stiffness. In Sec. $I V$ we study the finite temperature properties: susceptibility, correlation length and specific heat. In the last Section we comment on the implications of our results.

\section{QHRT EQUATION FOR THE $J_{1}-J_{2}$ MODEL}

We briefly summarize the basic steps followed for the derivation of the QHRT evolution equation. A detailed description of the method can be found in Ref ${ }^{20}$. Being interested in a parameter region where antiferromagnetic correlations dominate, it is convenient to include in the Hamiltonian a staggered external magnetic field which directly couples to the Néel order parameter. The Hamiltonian is then written in terms of a reference part $H_{0}$, which in our case just coincides with the external field, and the interaction term $H_{J}$, chosen as the Hamiltonian of the $J_{1}-J_{2}$ model

$$
H=H_{0}+H_{J}=-h \sum_{\mathbf{R}} e^{i \mathbf{g} \cdot \mathbf{R}} S_{\mathbf{R}}^{z}+H_{J}
$$

where $\mathbf{g}$ is the antiferromagnetic wavevector and $H_{J}$ is defined in (11). With our choice of $H_{0}$, the properties of the reference system are known. The method applies to all interactions $H_{J}$, assumed bilinear in some operator $\rho(r)$,i.e. $H_{J}=\frac{1}{2} \int d x d y \rho(x) w(x-y) \rho(y)$; in the present case $\rho(r)$ represents local spin variables and the interaction in Fourier space is $\tilde{w}(\mathbf{k})=2 J_{1}\left[\cos k_{x}+\right.$ $\left.\cos k_{y}\right]+4 J_{2} \cos k_{x} \cos k_{y}$. As a first step we write a formal perturbative expansion of the partition function of the model $Z=\operatorname{Tr} \exp (-\beta H)$ in powers of the interaction $\tilde{w}^{\underline{21}}$. Here we do not make use of any specific property of the reference system correlation functions 
but instead the diagrammatic perturbative expansion is carried out in full generality. Remarkably, the resulting perturbation series is formally equivalent to that of a classical system in $(2+1)$ dimensions, with the periodic "time" variable now belonging to the interval $(0, \beta)$ and the interactions described by the classical two body potential $w_{c}(\mathbf{r}, t)=w(\mathbf{r}) \delta(t) / \beta$. Mapping on classical systems are frequently employed to describe quantum models at phase transitions, where long wavelength fluctuations always dominate. However, we stress here that our approach does not rely on any continuum limit and preserves the microscopic character of the Hamiltonian: actually, the formal expansion of the partition function leads to a quantum-to-classical correspondence which is exact, valid in the whole temperature range and not limited to the critical regime.

The thermodynamics of the corresponding classical system can be studied within the Hierarchical Reference Theory (HRT) formalism ${ }^{22}$, successfully employed in the framework of classical statistical physics. HRT has been developed as a way to implement the basic ideas of the momentum space RG directly to microscopic classical Hamiltonians. The main goal of HRT is to describe how the inclusion of fluctuations over different length scales, affects the free energy of the model. For this purpose we define a sequence of auxiliary systems (named $Q$-systems) characterized by a cut-off dependent potential $\tilde{w}(k)_{Q}$ which coincides with the physically relevant one $\tilde{w}(k)$ for $k>Q$ and vanishes elsewhere ${ }^{23}$. Introducing a momentum cut-off in the interaction inhibits the antiferromagnetic fluctuations at long wavelengths, preempting the occurrence of phase transitions. When the cut-off $Q$ is reduced, the properties of the system continuously evolve between a mean field description, where fluctuations are absent, and those of the fully interacting system, where the fluctuations over all length scales are taken into account. Following this route, we obtain an exact set of differential equations expressing how the free energy $a^{Q}$ and the correlation functions change when the cut-off $Q$ is varied. In particular, the evolution of the Helmholtz free energy is given by

$$
\begin{aligned}
\frac{d a^{Q}}{d Q}= & \frac{1}{2 \beta} \sum_{\omega_{n}} \int_{\Sigma_{Q}} \frac{d^{2} k}{(2 \pi)^{2}}\left\{\ln \left(1-F_{Q}^{z z}\left(\mathbf{k}, \omega_{n}\right) \tilde{w}(\mathbf{k})\right)+\right. \\
& \left.\ln \left[\left(1+F_{Q}^{x x}\left(\mathbf{k}, \omega_{n}\right) \tilde{w}(\mathbf{k})\right)\left(1+F_{Q}^{x x}\left(\mathbf{k}+\mathbf{g}, \omega_{n}\right) \tilde{w}(\mathbf{k}+\mathbf{g})\right)+F_{Q}^{x y}\left(\mathbf{k}, \omega_{n}\right) F_{Q}^{x y}\left(\mathbf{k}+\mathbf{g}, \omega_{n}\right) \tilde{w}(\mathbf{k}) \tilde{w}(\mathbf{k}+\mathbf{g})\right]\right\}
\end{aligned}
$$

The summation is over the Matsubara frequencies $\omega_{n}=$ $2 \pi n / \beta$ and the integration is restricted to the one dimensional domain $\Sigma_{Q}$ defined by

$$
Q=\sqrt{1-\frac{1}{2}\left[\cos \left(k_{x}\right)^{2}+\cos \left(k_{y}\right)^{2}\right]}
$$

with $Q \in[0,1]$. Physically, the right hand side of this differential equation provides the contribution to the free energy due to fluctuations characterized by a wave vector belonging to the domain $\Sigma_{Q}$, which in fact spans the whole Brillouin zone when the cut-off parameter $Q$ varies from 1 to 0 . The choice of the sequence of surfaces is in principle arbitrary but definition (44) proves quite convenient because it respects the symmetries of the lattice, guarantees the stability of the partial differential equation (3) and leaves the critical fluctuations, of wave vector $\mathrm{g}$, at the end of the integration.

The key quantities appearing in Eq. (3) are the spinspin dynamical correlation functions of the $Q$-system $F_{Q}^{\alpha \beta}\left(\mathbf{k}, \omega_{n}\right)=<S^{\alpha}\left(\mathbf{k}, \omega_{n}\right) S^{\beta}\left(-\mathbf{k},-\omega_{n}\right)>$. When $Q=1$ no fluctuations have been included yet and the spin-spin correlation functions reduce to their expressions in meanfield approximation, obtained by keeping only the zero- loop contributions in the diagrammatic expansion:

$$
\begin{aligned}
& F_{Q=1}^{x x}(\mathbf{k}, \omega)=\frac{\mu_{\perp}+\tilde{w}(\mathbf{k}+\mathbf{g})}{m^{-2} \omega^{2}+\left(\mu_{\perp}+\tilde{w}(\mathbf{k}+\mathbf{g})\right)\left(\mu_{\perp}+\tilde{w}(\mathbf{k})\right)} \\
& F_{Q=1}^{x y}(\mathbf{k}, \omega)=\frac{m^{-1} \omega}{m^{-2} \omega^{2}+\left(\mu_{\perp}+\tilde{w}(\mathbf{k}+\mathbf{g})\right)\left(\mu_{\perp}+\tilde{w}(\mathbf{k})\right)} \\
& F_{Q=1}^{z z}(\mathbf{k}, \omega)=\frac{\delta_{\omega, 0}}{\mu_{\|}+\tilde{w}(\mathbf{k})}
\end{aligned}
$$

where $\mu_{\perp}$ and $\mu_{\|}$are known functions of temperature $T$ and staggered magnetization $m$. For $S=1 / 2: \mu_{\perp}=$ $2 T m^{-1} \tanh ^{-1}(2 m)$ and $\mu_{\|}=4 T\left(1-4 m^{2}\right)^{-1}$. The mean field form (5) of the correlation functions corresponds, after Wick rotation, to the single-mode approximation for the dynamic structure factor

$$
\operatorname{Im} S_{x x}(\mathbf{k}, \omega)=\frac{\omega}{1-e^{-\beta \omega}} \frac{\delta\left(\omega-\epsilon_{\mathbf{k}}\right)+\delta\left(\omega+\epsilon_{\mathbf{k}}\right)}{\mu_{\perp}+\tilde{w}(\mathbf{k})}
$$

where the quasi-particle dispersion $\epsilon_{\mathbf{k}}$ is given by

$$
\epsilon_{\mathbf{k}}=m \sqrt{\left(\tilde{w}(\mathbf{k})+\mu_{\perp}\right)\left(\tilde{w}(\mathbf{k}+\mathbf{g})+\mu_{\perp}\right)} .
$$

As pointed out in ${ }^{20}$, a better parameterization of the spin-spin dynamical correlation function (5) is in principle possible, allowing more complex dispersion relations. 
Here, for simplicity and in order to verify the reliability of the method for frustrated systems, we consider the simpler approximation reproducing the lowest order in SWT. Linearization of Eq. (7) around $\mathbf{k}=0$ shows that in mean field approximation, the spin velocity $c_{s}$ is proportional to the staggered magnetization $m$ : $c_{s}=m J_{1} \sqrt{8(1-2 \alpha)}$, which correctly reproduces only the zeroth order SWT.

For a generic value of the cut-off $Q \neq 1, F_{Q}^{\alpha \beta}(\mathbf{k}, \omega)$ are not known because they already include the contribution of short range correlations. As a consequence, equation (3) cannot be solved by straightforward integration and additional information on the form of the dynamical correlation functions is required. One possibility is to write an infinite set of exact differential equations also for the many particle correlation functions, thereby generating a full hierarchy of evolution equations: the QHRT. However, it is more convenient to limit our attention to the first equation of such a hierarchy, i.e. to Eq. (3), attempting a closure by imposing a suitable approximate parametrization of the spin-spin correlation function. The simplest choice, which we will adopt in this work, is to retain the same analytical structure of the mean-field approximation (15) keeping $\mu_{\perp}$ and $\mu_{\|}$as free, cut-off dependent parameters. Their initial value at $Q=1$ is set by the mean field expressions, while at $Q \neq 1$ they are obtained by use of the exact sum rules which relate the dynamical correlation functions to derivatives of the free energy $a^{Q}$ :

$$
\begin{gathered}
{\left[\frac{\partial a^{Q}}{\partial m}\right]^{-1} m=F_{Q}^{x x}(\mathbf{g}, \omega=0)=\left[\mu_{\perp}^{Q}-4\left(J_{1}-J_{2}\right)\right]^{-1}} \\
{\left[\frac{\partial^{2} a^{Q}}{\partial m^{2}}\right]^{-1}=F_{Q}^{z z}(\mathbf{g}, \omega=0)=\left[\mu_{\|}^{Q}-4\left(J_{1}-J_{2}\right)\right]^{-1}}
\end{gathered}
$$

Within such an approximate closure, the inclusion of fluctuations does not change the analytical structure of $F_{Q}^{\alpha \beta}$ : fluctuations lead to a renormalization of the parameters $\mu_{\perp}^{Q}$ and $\mu_{\|}^{Q}$ but do not allow for the creation of an incoherent contribution to the dynamical structure factor (6). By use of the representation (5) in the exact evolution equation (3) and summing over the Matsubara frequencies, we obtain an approximate, closed evolution equation for the free energy $a^{Q}$ as a function of the cut-off $Q$ and of the staggered magnetization $m$ :

$$
\frac{d a^{Q}}{d Q}=\frac{1}{2 \beta} \int_{\Sigma_{Q}} \frac{d^{2} p}{(2 \pi)^{2}} 4 \ln \left[\frac{\sinh \left(\frac{1}{2} \beta m \mu_{\perp}\right)}{\sinh \left(\frac{1}{2} \beta m \sqrt{\left(\mu_{\perp}+\tilde{w}(\mathbf{p})\right)\left(\mu_{\perp}+\tilde{w}(\mathbf{p}+\mathbf{g})\right)}\right)}\right]+\ln \left[\frac{\mu_{\|}^{2}}{\left(\mu_{\|}+\tilde{w}(\mathbf{p})\right)\left(\mu_{\|}+\tilde{w}(\mathbf{p}+\mathbf{g})\right)}\right]
$$

Equation (10), together with the thermodynamic consistency relations (89) and the mean field initial condition at $Q=1$, has been solved numerically for different values of the temperature $T$ and spin $S$. The $Q$-dependence of the parameters $\mu_{\perp}$ and $\mu_{\|}$is understood in Eq. (10). We recall that $Q$ is just a control parameter which allows for a gradual introduction of magnetic fluctuations: All physical quantities we are interested in refer to the $Q=0$ limit. However, the numerical determination of the free energy at $Q=0$ requires the integration of the parabolic partial differential equation in the whole interval $0<Q<1$. All the numerical results shown in the following sections will refer to $Q=0$. In order to simplify the notation, the label $Q$ will be dropped when we refer to $Q=0$. We observe that the form of equation (10) is unaffected by $S$ and the spin value just enters through the initial condition at $Q=1$. Equation (10) has been written in quasi-linear form and solved by a fully implicit finite difference scheme. We stress that Eq. (10) has been solved only at non-zero temperature and we did not attempt a direct analysis of the limiting equation as $T \rightarrow 0$.

\section{ZERO TEMPERATURE LIMIT}

We first investigate which information on the ground state properties of the frustrated antiferromagnet may be extracted form the QHRT formalism. Let us consider the behavior of the zero field staggered magnetization when fluctuations are included. By differentiating Eq. (10) with respect to the magnetization it is possible to obtain an evolution equation for the staggered field $h_{Q}(m)$, defined as the derivative of the free energy $a^{Q}$ with respect to the staggered magnetization, at fixed $m$. This quantity implicitly describes the dependence of the staggered magnetization $m_{Q}$ on the cut-off $Q$ at fixed magnetic field $h$ through the identity $h_{Q}\left(m_{Q}\right)=h$. Note that in mean field approximation the model displays a finite temperature critical point between a high temperature paramag-

\begin{tabular}{|c|c|c|c|c|c|c|}
\hline$\alpha=J_{2} / J_{1}$ & 0 & 0.1 & 0.2 & 0.3 & 0.35 & 0.4 \\
\hline$m_{0}(S=1 / 2)$ & 0.35 & 0.34 & 0.30 & 0.25 & 0.18 & 0.05 \\
\hline$m_{0}(S=1)$ & 0.84 & 0.81 & 0.75 & 0.64 & 0.52 & 0.34 \\
\hline
\end{tabular}

TABLE I: Ground state staggered magnetization $m_{0}$ for $S=$ $1 / 2$ (top panel) and $S=1$ (bottom panel). 


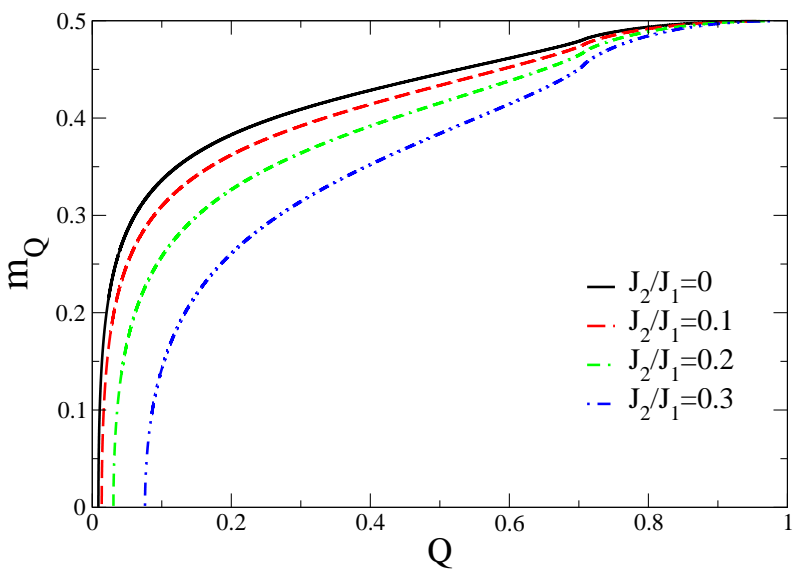

FIG. 1: $Q$-evolution of the zero field staggered magnetization for $S=1 / 2$ and different values of the frustration, at $T=$ $0.1 J_{1}$.

net and an antiferromagnetic ground state. As a consequence, the initial condition of our QHRT equation shows non vanishing spontaneous magnetization $m_{Q=1} \neq 0$ for $h=0$. According to the Mermin-Wagner theorem, thermal fluctuations depress, and eventually kill, long range Néel order and, as we know, QHRT does conform to this scenario 20 . Therefore, we expect that, at low temperature, the $Q$ evolution of the staggered magnetization in zero field is characterized by a smooth initial behavior which extrapolates to the $T=0$ order parameter, followed by a sudden drop close to $Q=0$ due to the action of long wavelength fluctuations. This expected qualitative behavior can be checked on the numerical solution shown in Fig. 1] Note that the QHRT equations preserve the convexity of the free energy in the physical limit $Q=0$ : This implies that at all temperatures $T \neq 0$, the zero-field inverse susceptibility $\chi^{-1}$ must be strictly positive at $Q=0$ and then it has to $\operatorname{cross} \chi_{Q}^{-1}=0$ at a non vanishing cut-off $Q^{*} \neq 0$. As a consequence, also the spontaneous magnetization, present at mean field level even at $T \neq 0$, vanishes at $Q^{*} \neq 0$ as shown in Fig. [1. The main effect of frustration on the evolution of the spontaneous magnetization is in fact to move this characteristic cut-off toward larger values due to the expected decrease of the correlation length.

Our estimates of the ground state staggered magnetization, based on Fig. 1 are reported in Table @ for both $S=1 / 2$ and $S=1$. The same data are shown in Fig. 2] together with the first and second order spin wave theory results ${ }^{24}$. As already discussed in Ref ${ }^{20}$, at $\alpha=0$ and $S=1 / 2$ the QHRT estimate is very close to the commonly accepted value $m_{0} \sim 0.307 \stackrel{25}{2}$. However, while first order SWT predicts a vanishing magnetization at $\alpha_{c} \sim 0.38$, our results indicate that the ground state magnetization extrapolates to zero only at significantly larger values of $\alpha \sim 0.43$.

At zero temperature, in a symmetry broken phase, the

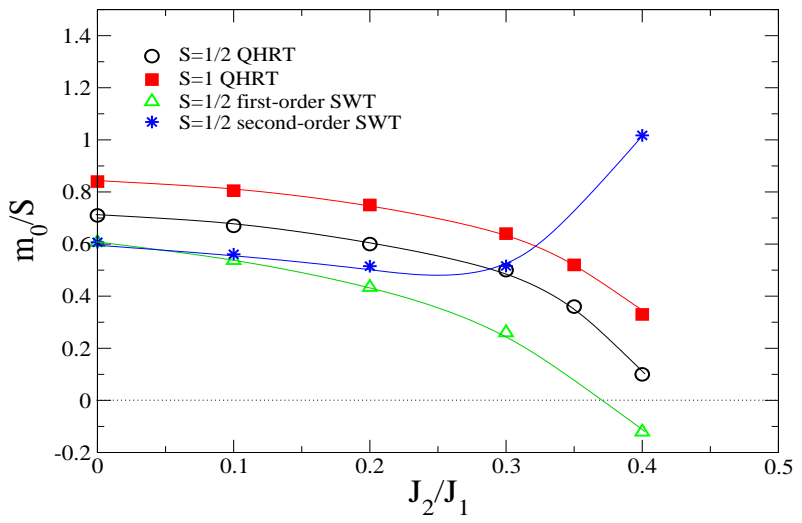

FIG. 2: Extrapolated ground state staggered magnetization $m_{0}$ divided by its classical value $S$. QHRT results for $S=1 / 2$ (empty circles) and $S=1$ (full squares). First-order (empty triangles) and second-order (stars) SWT results from ${ }^{24}$. Lines are a guide for the eyes.

spin stiffness $\rho_{s}$ can be defined in terms of the small momentum behavior of the transverse, static structure factor:

$$
S_{\perp}(k) \rightarrow \frac{\rho_{s}}{2 c_{s}} k
$$

Within QHRT, the spin velocity $c_{s}$ has been previously evaluated from Eq. (17) while $S_{\perp}(k)$ is easily obtained from the parametrization (5). As a result we obtain the simple analytical relationship: $\rho_{s}=m_{0}^{2}\left(J_{1}-2 J_{2}\right)$ which, by use of Tab. \ provides an estimate of the stiffness. The proportionality between $\rho_{s}$ and $m_{0}^{2}$ guarantees that at the quantum critical point, where the order parameter $m_{0}$ vanishes, the spin stiffness necessarily tends to zero, as expected.

\section{TEMPERATURE DEPENDENCE OF PHYSICAL PROPERTIES}

According to the definition of the spin-spin correlation function, we can define the staggered magnetic susceptibility in terms of the zero frequency value of $F^{\alpha \beta}(\mathbf{k}, \omega)$ at $\mathbf{k}=\mathbf{g}$

$$
\begin{aligned}
\chi_{\perp}^{s} & =F^{x x}(\mathbf{g}, 0)=\left(\mu_{\perp}-4\left(J_{1}-J_{2}\right)\right)^{-1} \\
\chi_{\|}^{s} & =F^{z z}(\mathbf{g}, 0)=\left(\mu_{\|}-4\left(J_{1}-J_{2}\right)\right)^{-1} .
\end{aligned}
$$

The results at $m=0$ are plotted in Fig. B as a function of temperature for several values of the frustration ratio. In agreement with Mermin-Wagner theorem, true long-range order is absent at any finite temperature in two dimensions and in fact the susceptibility obtained via QHRT remains finite at every $T \neq 0$. Conversely, the divergence of $\chi$ when zero temperature is approached is consistent with the onset of long range antiferromagnetic order in the ground state of the model. In order to better 


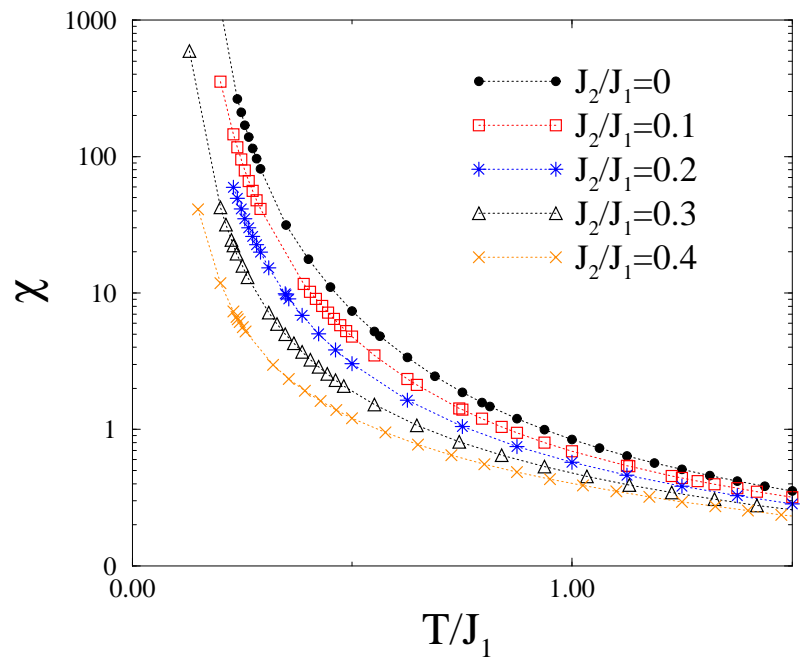

FIG. 3: $S=1 / 2$ low temperature staggered susceptibility versus temperature, for various values of the frustration at $m=0\left(\chi_{\perp}^{s}=\chi_{\|}^{s}\right)$. Lines are guides for the eyes.

analyze such a divergence we will refer to a closely related quantity: the antiferromagnetic correlation length, $\xi$ defined as $\left|\mathbf{k}_{P}\right|^{-1}$, where $\mathbf{k}=\mathbf{g}+i \mathbf{k}_{P}$ is the location of the poles of the function $F^{\alpha \beta}(\mathbf{k}, \omega=0)$. The same $\xi$ essentially governs the exponential decay of the equal-time correlation function at $T \neq 0$. Close to the critical value of the wave vector $\mathbf{k}=\mathbf{g}$, the two-point correlation function at $\mathbf{k}=\mathbf{g}+\mathbf{q}$ and $\omega=0$ takes the following form

$$
F^{\alpha \beta}(\mathbf{g}+\mathbf{q}, 0) \sim \frac{1}{\mu-4\left(J_{1}-J_{2}\right)+q^{2}\left(J_{1}-2 J_{2}\right)}
$$

leading to a correlation length algebraically related to the susceptibility (12) and simply expressed as a function of the parameter $\mu$ :

$$
\xi^{-2}=\frac{\mu-4\left(J_{1}-J_{2}\right)}{J_{1}-2 J_{2}}=\frac{1}{\chi^{s}\left(J_{1}-2 J_{2}\right)}
$$

The numerical values of $\xi(T)$ can be easily deduced from Fig. 3 and clearly show a strong suppression of long range correlations when the frustration is increased. As predicted by weak-coupling (one-loop) RG analysis of the usual NLSM ${ }^{26}$, when the temperature is lowered the system enters the classical renormalized regime characterized by an exponential divergence of $\xi$. In this region of the phase diagram the correlation length diverges exponentially, just as it would in the classical model:

$$
\xi \sim \exp \left(2 \pi \tilde{\rho}_{s} / T\right)
$$

where the coefficient $\tilde{\rho}_{s}$ is identified through the weak coupling RG analysis, with the spin-stiffness renormalized by quantum fluctuations $16,27.28$. As shown in Fig. (4), the QHRT equation well reproduces, for different

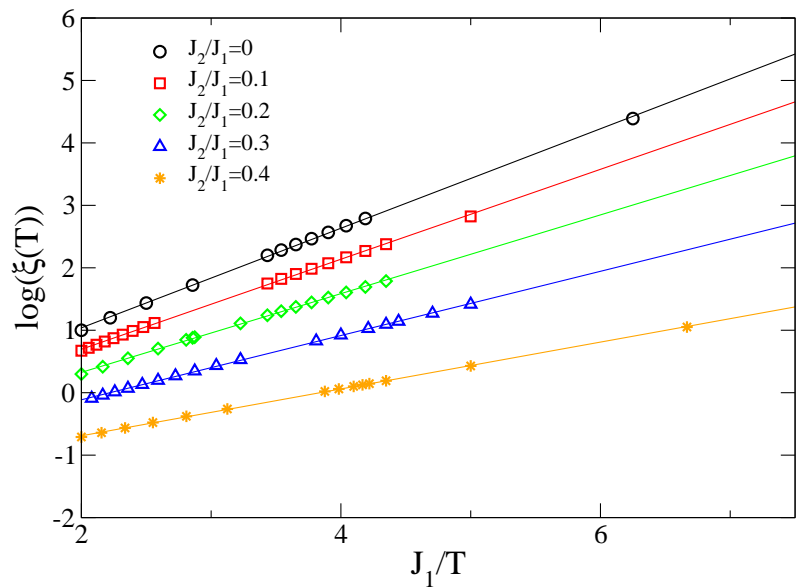

FIG. 4: $\log (\xi(T))$ versus the inverse temperature $J_{1} / T$ for the $S=1 / 2$ system and different values of the frustration ratio. Straight lines are linear fits of the low temperature data, which clearly follow the exponential law (15). Qualitatively similar results have been obtained for $S=1$.

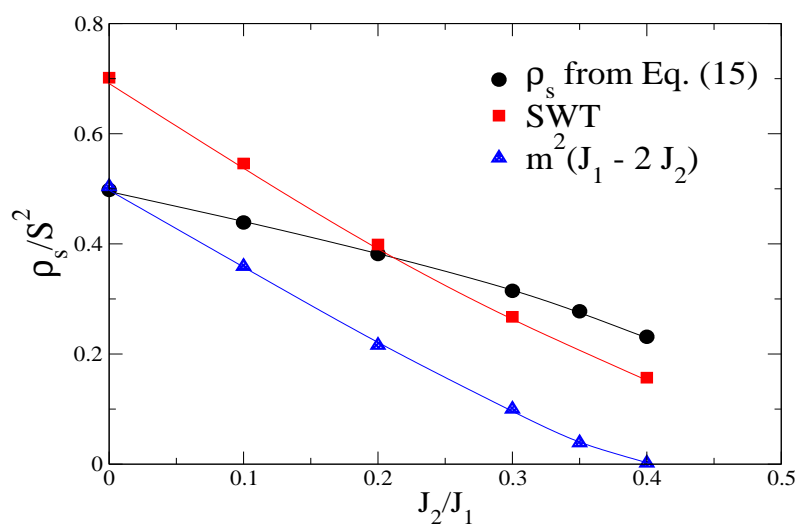

FIG. 5: $\quad S=1 / 2$ renormalized spin stiffness as a function of the frustration. We report the values obtained from Eq. (11) (triangles), the coefficient $\tilde{\rho}_{s}$ from the low temperature divergence of the correlation length, Eq. (15) (circles) and the SWT estimate (squares) from 16 .

values of the frustration, the exponential behavior of $\xi$ at low temperature as already found in Ref 20 . Eq. 15] is valid in the low temperature limit $(T<<J)$ when correlations at the antiferromagnetic wave vector become long ranged. In this regime it is possible to estimate the renormalized stiffness coefficient $\tilde{\rho}_{s}$ which governs the exponential divergence of the correlation length (15) via a linear fit of the data in Fig. [4

The results of such a fit are shown in Fig. [5 together with the direct evaluation of the stiffness $\rho_{s}$ via Eq. (11) at $S=1 / 2$. Frustration depresses the correlation length, nevertheless $\tilde{\rho}_{s}$ does not vanish in the range of frustration $[0,0.4]$ we have investigated and no clear sign of quantum critical point can be inferred from these data. A simi- 
lar result was actually found in Ref ${ }^{16}$ using variational spin-wave approach while exact diagonalization studies, followed by finite size scaling,, 9.29 are not conclusive, the spin stiffness estimate depending on the chosen cluster geometry.

The comparison between $\tilde{\rho}_{s}$ and the direct evaluation of the stiffness $\rho_{s}$ via Eq. (11) (see Fig. 15) clearly shows that, within QHRT, the exponential divergence of the correlation length at low temperature is not governed by the same quantity which appears in the zero temperature transverse structure factor, contrary to the prediction of the $\mathrm{RG}$ analysis of the non linear sigma mode ${ }^{30}$. In order to investigate the origin of such a discrepancy, we performed a careful analysis of the QHRT equation in the classical limit at $J_{2}=0$, which corresponds to the $S \rightarrow \infty$ case. At zero temperature, the ground state magnetization is clearly $m_{0}=S$, providing the correct limiting result for the classical spin stiffness: $\rho_{s}=S^{2} J_{1}$. Instead, the numerical integration of the evolution equation gives a diverging correlation length consistent with Eq. (15) where the renormalized stiffness is given by $\tilde{\rho}_{s}=S^{2} J_{1} / 3$. This estimate actually coincides with the lowest order term of a large $N$ expansion in the $\mathrm{SU}(N)$ non linear sigma mode 28 evaluated at $N=3$. The correct RG result for the $\mathrm{SU}(N)$ non linear sigma model, valid at all orders in $N$, is instead given by $\tilde{\rho}_{s}=S^{2} J_{1} /(N-2)$ which, at $N=3$ coincides with the zero temperature evaluation of $\rho_{s}$. A careful analysis of the RG approach in $2+\epsilon$ dimension ${ }^{30}$ suggests that the origin of the discrepancy found in the QHRT equations may be traced back to the chosen parametrization of the correlation functions (5). The reason is twofold:

- In the QHRT equation (10) both longitudinal and transverse correlations contribute to one loop order, while in the RG approach only the transverse propagator enters the beta-function. Due to the non linear sigma model constraint, at low temperature the longitudinal correlations can be expressed in terms of the transverse ones:

$$
\begin{aligned}
F^{z z}(r) & \sim\left[<\left[1-\pi(r)^{2}\right]^{\frac{1}{2}}\left[1-\pi(0)^{2}\right]^{\frac{1}{2}}>-\right. \\
& \left.-<\left[1-\pi(0)^{2}\right]^{\frac{1}{2}}>^{2}\right] \\
& \sim \frac{1}{4}\left[<\pi(r)^{2} \pi(0)^{2}>-<\pi(0)^{2}>^{2}\right] \\
& \sim \frac{1}{2}\left[F^{x x}(r)\right]^{2}
\end{aligned}
$$

where $\pi(r)$ identifies transverse spin fluctuations.

- Moreover, within our approximation (5) the wavevector dependence of the correlation function is not renormalized by fluctuations, while the RG analysis in $2+\epsilon$ dimensions shows that the one loop corrections do affect the momentum dependence of the one particle irreducible two point function ${ }^{30}$ :

$$
\Gamma(p)=\frac{\Lambda^{\epsilon}}{T}\left[p^{2}\left(1+\frac{T}{2 \pi \epsilon}\right)+h\left(1+\frac{(N-1) T}{4 \pi \epsilon}\right)\right]
$$

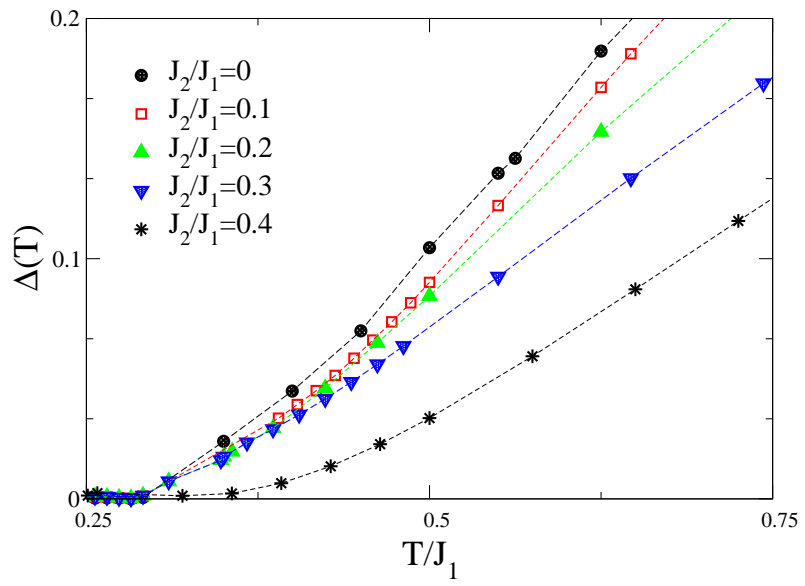

FIG. 6: Plot of the function $\Delta(T)$ defined as $\Delta(T)=$ $\left|\log (\xi(T))-l_{f i t}(T)\right|$, where $l_{f i t}(T)$ is the linear fit function describing the asymptotic behavior of $\log \xi(T)$ at low $T$. The function is plotted for different value of the frustration ratio.

where $\Lambda$ is the ultraviolet cut-off of the $O(N)$ non linear sigma model. This expression should be compared with the long wavelength limit of the corresponding QHRT approximation:

$$
\Gamma(\mathbf{p})=\beta \mu_{\perp}+\beta \tilde{w}(\mathbf{p}+\mathbf{g})
$$

showing that in QHRT the momentum dependence is fixed by the spin-spin interaction.

These remarks suggest that a more flexible parametrization of the spin correlations should be used in the QHRT equations in order to achieve a quantitative representation of the low temperature divergence of the correlation length.

In order to better understand the effects of frustration on the onset of Néel order, we also investigated the $\alpha$ dependence of the temperature $T_{\times}$below which Eq. (15) is valid. In the following we refer to $T_{\times}$as crossover temperature. At mean-field level we known that the inclusion of a n.n.n. interaction increases the bare value of the coupling constant $g$ and tends to destroy the Néel order. When the system approaches the quantum critical point, controlled by the $g=g_{c}$ fixed point, the RG picture (qualitatively) predicts a decrease of the crossover temperature. The crossover temperature can be estimated by studying the temperature dependence of the function $\Delta(T)$ defined as $\Delta(T)=\left|\log (\xi(T))-l_{f i t}(T)\right|$, where $l_{f i t}(T)$ is the linear fit function describing the asymptotic behavior of $\log \xi(T)$ at low $T: \Delta(T)$ then measures the deviations of $\xi(T)$ from the exponential law (15). Figure [6 clearly shows that it is possible to identify a crossover temperature $T_{\times}$by the requirement $\Delta(T) \approx 0$ for $T<T_{\times}$. The results reported in Fig. 6 seem to indicate that $T_{\times}$does not depend much on $\alpha$, its value remaining basically unchanged when frustration is increased. The weak dependence of the crossover temperature on frustration may suggest either a very narrow crit- 


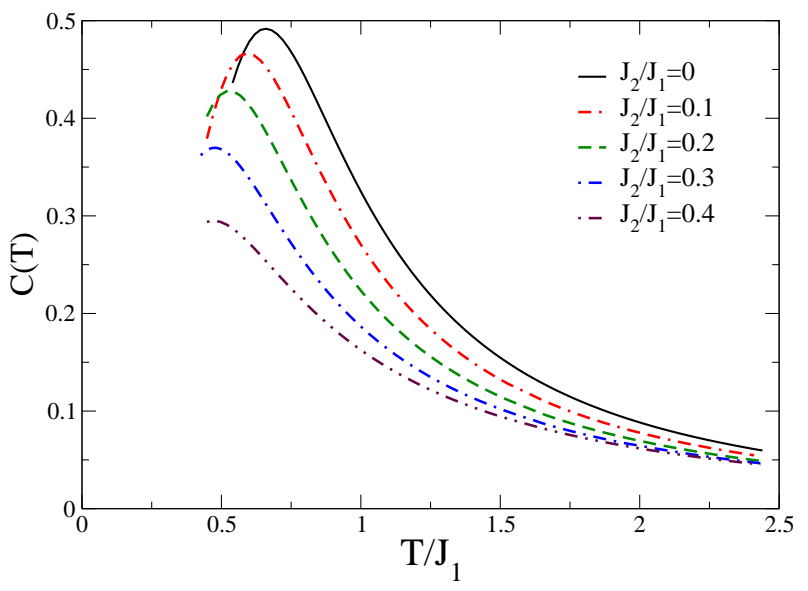

FIG. 7: Zero field specific heat for $S=1 / 2$

ical region near the quantum transition, or a shift in the quantum critical point toward larger values of $\alpha>0.5 \frac{16}{}$.

Finally, from the free energy we calculated zero field specific heat $C(T)$ for $S=1 / 2$ and different frustrations, shown in Fig. (7). As expected, frustration play a minor role at high temperature, the specific heat showing a paramagnetic behavior as $1 / T^{2}$ for all values of the coupling $\mathrm{J}_{2}$. The position of the maximum moves at low temperatures and its intensity decreases for increasing value of the frustration, in agreement with SWT 16 and small-size Exact Diagonalization results 33 .

\section{CONCLUSIONS}

We have studied finite temperature properties of the frustrated Heisenberg model on a square lattice in the Néel phase, by use of the quantum hierarchical reference theory, a non-perturbative implementation of the renormalization group method. We derived an exact evolution equation which describes how the free energy of the system changes when antiferromagnetic fluctuations over growing wavelengths are included. This equation is supplemented by an approximate representation of the dynamical correlation functions of the model (5). Within the limitations induced by our parameterization of the two-point correlations, the method employed does not suffer from uncertainties in the extrapolation to the thermodynamic limit ${ }^{31}$. Our results are valid in a wide range of temperature and for all spin values because our approach does not rely on any mapping on effective long wavelength theory and preserves the microscopic features of the model Hamiltonian. We focused on the role of fluctuations of wavevector close to $\mathbf{g}=(\pi, \pi)$ and then we could not investigate the possible occurrence of other order parameters characterized by different periodicities, as in spiral or collinear phases.

Starting from the knowledge of the free energy of the system, we calculated spin susceptibility, two point correlation function and correlation length at finite temperature and we also estimated ground state properties such as spin stiffness and staggered magnetization by extrapolation to the $T \rightarrow 0$ limit. Calculations have been performed for spin $S=1 / 2$ and $S=1$ and different values of the frustration ratio, in order to investigate the role and extent of quantum corrections on top of the semiclassical spin-wave picture. The investigated range of frustration is restricted to $\alpha<0.4$ because beyond this point, fluctuations at wavevector $(\pi, 0)$ are expected to become important and our simple parametrization of the dynamical correlation functions cannot be retained.

The spontaneous staggered magnetization extrapolates to zero at about $\alpha \sim 0.43$ for $S=1 / 2$ while a stronger frustration is required for $S=1$. Within the QHRT approach, both the spin velocity and stiffness are related to the order parameter and therefore are expected to vanish at the quantum critical point. For comparison we recall that in the non linear sigma model representation of the quantum antiferromagnets, when the $T=0$ critical point is approached, both the staggered magnetization and the spin stiffness vanish, while the spin velocity is expected to remain finite. Our results indicate that frustration strongly suppresses the correlations at finite temperature but, at the same time, the value of the crossover temperature $T_{\times}$remains basically unchanged when the frustration is increased. This fact may suggest a very narrow critical region near the quantum transition or a shift of the quantum critical point toward larger values of $\alpha>0.5$. The estimate of the renormalized stiffness $\tilde{\rho}_{s}$ obtained via the low temperature divergence of the correlation length, Eq. (15), does not vanish in the whole frustration range we investigated and is remarkably larger than the direct evaluation of the stiffness $\rho_{s}$ obtained through the zero temperature static structure factor (11). The equality of these two quantities is commonly expected on the basis of the known behavior of the $\mathrm{SU}(N)$ sigma-model in the $N \rightarrow \infty$ limit and the $\mathrm{RG}$ analysis in $2+\epsilon$ dimension ${ }^{30}$. The difference in the two estimates of $\rho_{s}$ we found in the $\mathrm{SU}(2)$ case is most likely a consequence of the approximate closure (15) we have studied, as shown by a detailed analysis of the classical limit of the QHRT equations. In order to go beyond the approximation examined in this work, a representation of the low temperature spin correlations richer than those commonly adopted in the many body literature ${ }^{32}$ is needed.

Nowadays, real materials displaying the properties of the $J_{1}-J_{2}$ model in the Néel phase have not been yet synthesized. Nevertheless, the recent experiments at high pressure performed on the $\mathrm{Li}_{2} \mathrm{VOSiO}_{4}{ }^{34}$ seem to indicate that a wide range of the $J_{1}-J_{2}$ phase diagram can be studied by applying high hydrostatic pressure, which modifies the frustration ratio $J_{2} / J_{1}$ by changing the bond lengths and angles. Although the relationship between pressure and frustration ratio is not obvious at all, we believe that the results of our investigation may 
be useful for the interpretation of future neutron scattering or NMR-NQR experiments. In addition ground state configuration of the $\mathrm{VOMoO}_{4}$ compound is still a controversial issue, first principle calculation of the $\mathrm{J}_{2}$ coupling being in disagreement with the estimates based on the NMR-NQR experiments. Specific heat data $\stackrel{34}{\text { for }}$ the $\mathrm{VOMoO}_{4}$ compound may be analyzed on the basis of our finite-temperature results, the position of the maximum in the zero-field specific heat giving an alternative way for estimating the frustration parameter.

We thank L. Reatto and P. Carretta for useful discussions. The financial support of MIUR through PRIN2003 is also acknowledged.
1 A. Harrison J.Phys. Condens. Matter 16 553-572 (2004); H. Kageyama et al. Physica B 3291020 (2003); H. Kageyama, K. Yoshimura, R. Stern, N.V. Mushnikov, K. Onizuka, M. Kato, K. Kosuge, C.P. Slichter, T. Goto and Y. Ueda Phys. Rev. Lett 82, 3168 (1999).

2 R.Melzi, P.Carretta, M.Mambrini, M.Troyer,P.Millet and F.Mila Phys. Rev. Lett. 85,1318 (2000); R.Melzi, S.Aldrovaldi, F.Tedoldi, P. Carretta, P. Millet and F. Mila Phys. Rev. B 64, 024409 (2001).

3 For a review see: G.Misguich and C.Lhuillier in "Frustrated spin systems", edited by H.T.Diep, World-Scientific (2003).

4 M.Inui,S.Doniah, M.Gabay Phys. Rev. B 38,6631 (1988).

5 P.Carretta, R.Melzi, N.Papinutto, P.Millet Phys. Rev. Lett. 88, 47601 (2002); P.Carretta, N. Papinutto, C.B. Azzoni, M.C. Mozzati, E. Pavarini, S. Gonthier and P.Millet Phys. Rev. B 66,094420 (2002).

6 See for instance: V.N. Kotov et al., Phylos. Mag. B 80, 1483 (2000); L. Capriotti, Int. J. Mod. Phys. B 15, 1799 (2001).

7 S.Sorella Phys. Rev. Lett. 80, 4558 (1998); S.B.Fahy and D.R.Hamann Phys. Rev. Lett. 65, 3437 (1990); Phys.Rev.B 43, 765 (1991).

8 S.Bacci, E. Gagliano, E. DagottoPhys.Rev.B 44, 285 (1991).

9 A.E.Feiguin, C.J. Gazza, A.E. Trumper, H.A. Ceccatto Phys.Rev.B 52,15043 (1995).

10 T.Einarsson and H.J. Schulz Phys.Rev.B 51, 6151 (1995).

11 J.Bonca, J.P. Rodriguez, J. Ferrer, K.S. Bedell Phys. Rev. B 50, 3415 (1994).

12 See for instance G.S.Rushbrooke, G.A.Baker, Jr., and P.J.Wood, in Phase Transition and Critical Phenomena, vol. 3, C.Domb and M.S.Green eds. (Academic Press, London, 1972)

13 R.R.P.Singh, W. Zheng, J.Oitmaa, O.P. Sushkov, C.J. Hamer Phys. Rev. Lett. 91,017201 (2003).

14 G.Misguich, B. Bernu, L. Pierre Phys. Rev. B 68, 113409 (2003).

15 P.Chandra and B.Doucot Phys. Rev. B 38, R9335 (1988).
16 N.E. Ivanov and P. Ch. Ivanov Phys.Rev.B 46, 8206 (1992).

17 F. Mila, D. Poilblanc, C. Bruder Phys. Rev. B 43, 7891 (1991).

18 L. Capriotti, A. Fubini, T. Roscilde, V. Tognetti Phys. Rev. Lett. 92, 157202 (2004);C.Weber, L.Capriotti, G.Misguich, M. Elhajal and F.Mila Phys. Rev. Lett 91, 177202 (2003).

19 I.Affleck in Field theory Methods and Quantum Critical Phenomena, proceedings of the XILX Les Houches School, E.Brézin and J.Zinn-Justin (1988).

20 P.Gianinetti and A.Parola Phys.Rev.B 63, 104414 (2001).

21 L.Fetter and J.D.Walecka Quantum Theory of Many particles systems (MCGraw-Hill, New York, 1971).

22 A.Parola and L.Reatto Adv. Phys.44, 211 (1995).

23 Here, for simplicity, we consider spatially isotropic systems. The correspondig expressions appropriate for a lattice model will be given later.

24 J.Igarachi J. Phys. Soc. Jap 62, 4449 (1993).

25 B.B. Beard et al. Phys.Rev. Lett. 80, 1742 (1998); J.K. Kim and M. Troyer, Phys. Rev. B 80, 2705 (1998).

26 S.Chakravarty, B.I. Halperin and D.R Nelson Phys.Rev.B 39, 2344 (1989)

27 A.V.Chubukov, S. Sachdev, J. Ye Phys. Rev. B 49, 11919 (1994)

28 S. Sachdev Quantum Phase Transitions (Cambridge University Press, 1999)

${ }^{29}$ H.J.Schultz et al. J. Phys. I (France) 6, 675 (1996).

30 See for instance, J. Zinn-Justin Quantum field theory and critical phenomena (Clarendon Press, Oxford, 1989).

31 As pointed out in ${ }^{10}$, for a finite system even a disordered (spin liquid) phase may have a finite stiffness.

32 A. Auerbach Interacting Electrons and Quantum Magnetism (Springer Verlag, New York 1994).

33 S.Bacci, E. Gagliano and E. Dagotto Phys. Rev. B 44, 285 (1991).

34 P.Carretta et al. J.Phys.:Condens. Matter 16, 849-586 (2004).

35 P.Carretta private communication. 